\title{
SISTEMAS UNIVERSAIS PARA A AMÉRICA LATINA: JOVENS E ANTIGAS INOVAÇÕES NOS SERVIÇOS DE SAÚDE
}

\author{
Universal health systems for Latin America: recent and old innovations in health \\ services
}

\section{Sistemas universales para América Latina: jóvenes y antiguas innovaciones de los servicios de salud}

\author{
Eleonor Minho Conill \\ Observatório Iberoamericano de Políticas e Sistemas de Saúde - OIAPSS - Florianópolis (SC) - Brasil
}

\section{RESUMO}

Objetivo: O trabalho discute a necessidade de se equilibrar "antigas e jovens" inovações na construção de sistemas de saúde. Síntese dos dados: Descrição da trajetória dos sistemas contemporâneos com análise das reformas feitas no Brasil, Chile, Colômbia, Espanha e Portugal. Cinco momentos foram identificados: expansão dos serviços, reformas "democrático-racionalizadoras", ajustes neoliberais, reformas pró-coordenação, novos ajustes e proposta de cobertura universal para países de média e baixa renda. No Chile e na Colômbia houve a introdução explícita de seguros privados, no Brasil a implementação de um sistema público integrado sob financiamento fiscal. Nos três países a segmentação e a fragmentação dos serviços ainda predominam, com uma complexa composição de serviços públicos e privados. Portugal e Espanha implementaram sistemas nacionais que integram "antigas e jovens inovações", cuja macroeficiência tem sido demonstrada. Conclusão: Na América Latina, propostas de universalização da cobertura sem mudanças no modelo assistencial trazem riscos de um aumento desregulado de consumo sem correspondência com as necessidades de saúde da população. O conhecimento e o debate a partir das experiências acumuladas no Brasil e nos países ibéricos podem contribuir para o enfrentamento desses desafios.

Descritores: América Latina; Reforma dos Serviços de Saúde; Inovação.

\begin{abstract}
Objective: The paper discusses the need to balance "old and recent" innovations in the construction of health systems. Data synthesis: Description of the trajectory of contemporary systems with analysis of the reforms conducted in Brazil, Chile, Colombia, Spain and Portugal. Five moments were identified: expansion of services, "democratic-rationalizing" reforms, neoliberal adjustments, pro-coordination reforms, further adjustments and the proposal of universal coverage for low- and middle-income countries. In Chile and Colombia there was the explicit introduction of private insurance and, in Brazil, the implementation of an integrated public system under fiscal financing. In these three countries, segmentation and fragmentation of services still predominate, with a complex composition of public and private services. Portugal and Spain have implemented national systems integrating "old and recent innovations", whose macro-efficiency has been demonstrated. Conclusion: In Latin America, proposals to universalize coverage without changes in the health care model pose risks of a deregulated increase in consumption, not corresponding to the health needs of the population. Knowledge and debate based on the experiences accumulated in Brazil and in the Iberian countries can contribute to coping with these challenges.
\end{abstract}

Descriptors: Latin America; Health Care Reform; Innovation.

\section{RESUMEN}

Objetivo: El estudio discute la necesidad del equilibrio entre las "antiguas y jóvenes" innovaciones para la construcción de sistemas de salud. Síntesis de los datos: Descripción de la trayectoria de los sistemas contemporáneos con análisis de las

Este artigo foi selecionado, corrigido e aprovado pela Comissão Científica do Fórum Internacional de Sistemas Universais de Saúde, seguindo suas normas e formatação. 
reformas hechas en Brasil, Chile, Colombia, España y Portugal. Se identificaron cinco momentos: la expansión de los servicios, las reformas "democrático-racionalizadoras", los ajustes neoliberales, las reformas pro-coordinación, los nuevos ajustes y la propuesta de cobertura universal para los países de media y baja renta. En Chile y Colombia hubo la introducción explicita de los seguros privados y en Brasil la implementación de un sistema público integrado bajo financiación fiscal. En los tres países la segmentación y la fragmentación de los servicios aún predominan con una compleja composición de servicios públicos y privados. Portugal y España han implementado sistemas nacionales que integran "antiguas y jóvenes innovaciones" cuya macro eficiencia ha sido demostrada. Conclusión: En América Latina, las propuestas de universalización de la cobertura sin cambios en el modelo asistencial causan riesgos de un aumento desregulado del consumo sin correspondencia con las necesidades de salud de la población. El conocimiento y el debate a partir de las experiencias acumuladas en Brasil y en los países ibéricos pueden contribuir para el afrontamiento de eses desafíos.

Descriptores: América Latina; Reforma de la Atención de Salud; Innovación.

\section{INTRODUÇÃO}

Inovação e trabalho em rede são propostas que se tornaram frequentes na discussão sobre modos de organizar sistemas de saúde. Advém das transformações contemporâneas do sistema produtivo marcado pelos desafios de garantir a acumulação em mercados globalizados cada vez mais competitivos. Nas primeiras formulações sobre o tema, inovação compreendia tanto um novo produto como um novo processo de produção ou uma nova forma de organização. Na década de 1970, o conceito sofreu modificações passando a incorporar, também, uma nova idéia, uma nova prática ou um novo material. Atualmente, o conceito está associado à originalidade, a algo único, mas relevante e que não precisa ser, necessariamente, novo(1).

O princípio de equidade que fundamenta os sistemas universais torna necessário procurar modos de prestar serviços que garantam qualidade, bem-estar e sustentabilidade. Isso tem determinado a busca por maior racionalidade na organização dos serviços. Os componentes dessa racionalidade, denominadas "antigas inovações" são suficientemente conhecidos, pois vem sendo repetidos em discursos ou experimentados em algumas reformas há décadas. Incluem o trabalho com uma população ou grupos definidos, o desenvolvimento de vínculos com uma equipe de profissionais e a garantia de acesso a níveis de atenção coordenados para continuidade de um cuidado integral. Dentre esses conceitos, a estratégia de atenção primária (APS) é certamente o mais difundido. No entanto, mesmo que tenham sido demonstradas evidências da macroeficiência nos sistemas orientados por esses princípios $^{(2)}$, essas propostas aparecem e desaparecem da formulação de políticas e das reformas setoriais num movimento aparentemente pendular.

Já as "jovens" inovações correspondem a um grupo de medidas ou ações que se mostram importantes pela transição demográfica e epidemiológica e, principalmente, pela incorporação de novas tecnologias. As tecnologias de comunicação e informação (TICs) trazem ganhos de eficiência, facilitam o trabalho em rede, permitem um acompanhamento gerencial e tem um grande potencial para estabelecer novas relações entre usuários e os serviços. As que se referem aos processos de diagnóstico e terapia modificaram o trabalho em saúde e permitiram ganhos de sobrevida. Mas, também, é necessário controlar seu uso abusivo e inadequado o que levou ao surgimento do conceito de prevenção quaternária ${ }^{(3)} e$ a inclusão de questões relacionadas com a segurança do paciente nas avaliações da qualidade dos serviços.

O presente trabalho discute a necessidade de se equilibrar "antigas e jovens" inovações na construção de sistemas de saúde.

\section{SÍNTESE DOS DADOS}

O argumento central deste texto é de que é importante implementar "jovens inovações" sem perder de vista o grau de implantação das que se denominam "antigas", principalmente, no caso da expansão de direitos em saúde na América Latina. Inicialmente, serão descritas as principais características que marcaram as conjunturas dos sistemas de saúde, mostrando propostas centrais em cada um dos períodos analisados. Em seguida, será discutida a trajetória das reformas no Chile, na Colômbia e no Brasil por expressarem, ainda que de modo distinto, os discursos predominantes nessas conjunturas. Para finalizar, serão apontadas as ações realizadas no desenvolvimento dos sistemas nacionais da Espanha e Portugal que contribuíram de modo favorável ao seu desempenho, destacando elementos que parecem ser importantes em qualquer inovação no campo da saúde. 


\section{Trajetória dos sistemas de saúde contemporâneos e de suas reformas: um movimento pendular de "antigas inovações" no modo de organizar e prestar serviços?}

Embora o termo cobertura esteja associado ao estabelecimento de metas de programas de saúde pública, se refere, também, ao conjunto de serviços garantidos por sistemas ou seguros públicos e privados, através de regulamentação legal, regras regulatórias e/ou contratuais. Mas há uma grande diferença entre lei e direito, pois este implica num processo social mais amplo com criação de valores, normas e uma dinâmica social que garanta sua execução. A expressão operacional do direito a saúde também envolve uma extensa gama de possibilidades que vão desde um entendimento mais restrito de saúde até os diversos modos para organizar e prestar serviços para garantir a universalidade de serviços integrais. Na conquista de direitos há um embate de concepções e projetos que emergem de diferentes atores ou forças sociais e influenciam na abrangência e nas características das políticas públicas.

Uma das primeiras classificações de sistemas de saúde( ${ }^{(4)}$ coincide com a tipologia dos modelos de proteção social, pois o autor os dividia em assistência pública, seguros e serviços nacionais ${ }^{(5)}$. Na década de 1980 essa classificação foi alterada sugerindo-se a existência de três grandes formatos: o sistema empresarial-permissivo ou de mercado, no qual predomina o financiamento privado e a assistência pública, os seguros sociais públicos e os sistemas ou serviços nacionais. Os Estados Unidos representam a primeira modalidade, França e Alemanha são exemplos da segunda e, o Reino Unido, Canadá, Espanha e Portugal da terceira.

A conjuntura do pós-guerra com a criação da Organização das Nações Unidas (ONU) e a Declaração Universal dos Direitos Humanos impulsionou os Estados de Bem-Estar Social nas economias industrializadas européias (Welfare States) com um maior reconhecimento da importância das políticas redistributivas para a coesão social. $O$ surgimento do National Health Service (NHS) no Reino Unido é considerado um marco por seu caráter universal e características de prestação de serviços com cobertura integral e sem ônus financeiro direto. Em 1958, um seguro hospitalar é votado no Canadá ampliando-se em 1966 para um seguro universal e também integral ("all medically necessary") conhecido como Medicare.

Gradativamente, nos países que integram a Organization for Economic Co-Operation and Development (OECD) a cobertura dos seguros públicos se expandiu com uma maior inclusão e homogeneidade no rol de serviços. Por exemplo, uma lei de cobertura universal em vigência na França desde o ano 2000, incluiu a pequena parcela da população que ainda dependia da assistência pública. O Sistema Nacional de Salud (SNS) espanhol é o resultado da extensão da Asistencia Sanitária de la Seguridad Social (ASSS), cuja origem foi o seguro obrigatório de doença estabelecido nos anos 40 . As mudanças socioeconômicas haviam permitido o desenvolvimento considerável do sistema de proteção social que cobria mais de $80 \%$ da população com uma expansão de suas instalações e serviços. Em 1990 através do Decreto Real 1088/89, se completa uma universalização de facto com a inclusão da população sob a assistência pública ${ }^{(6,7)}$.

$\mathrm{Na}$ América Latina ainda persistem importantes desigualdades com grande heterogeneidade na cobertura e na prestação de serviços, sendo difícil classificar os sistemas a partir dessa tipologia. Em muitos países, seguros sociais convivem com a assistência prestada por serviços públicos ao restante da população, existindo poucos sistemas nacionais que propõem a universalização atenção (Cuba, Brasil, Costa Rica).

Embora tenha ocorrido uma expansão do acesso, principalmente aos serviços básicos de saúde, houve também um importante crescimento de seguros privados em todo continente latino-americano. Em países como Uruguai (Sistema Nacional Integrado de Salud), Venezuela (Sistema Público Nacional de Salud) e Equador, ocorreram esforços no sentido de ampliar a cobertura, unificar prestações melhorar a coordenação entre os serviços sob controle público. No Chile, na Colômbia e no Peru a estratégia para expansão do acesso implementou coberturas diferenciadas, com uma maior participação dos seguros privados no caso das reformas chilena e colombiana ${ }^{(8)}$. $O$ gasto público em saúde permanece baixo (US\$182/ habitante, 3,7\% do PIB) com elevado gasto direto das famílias ${ }^{(9)}$. Embora o gasto social tenha crescido na região (19,1\% do PIB em 2012-2013, em grande parte por programas de transferência de renda), o crescimento do gasto em saúde foi modesto passando de $3,2 \%$ pra $4,2 \%{ }^{(10)}$.

De um modo geral, o caminho percorrido na expansão de direitos nos sistemas de saúde contemporâneos não foi linear, sendo marcado por uma sucessão de ajustes e reformas. A análise dessa trajetória a partir da metade do século XX, permite sintetizar os seguintes momentos principais ${ }^{(6)}$ : 1950-1970 com expansão dos serviços centrados no modelo biomédico e na prestação de serviços hospitalares; Década de $1970 \mathrm{com}$ as reformas democráticoracionalizadoras como resposta ao aumento de gastos e demandas sociais. As propostas incluem medidas de ordem técnica e gerencial com ações inovadoras e de racionalização das práticas (comunidade como fundamento das necessidades de saúde, hierarquização, coordenação e integração do cuidado) e, também propostas participativas 
com inovações nas relações políticas sugerindo uma maior democratização dos serviços (mudanças na divisão do saber/poder com ampliação das equipes, incorporação de agentes da comunidade, acesso e controle social).

É nesse contexto que a estratégia de atenção primária emerge como consenso mundial para o alcance dos direito a saúde, o que foi sintetizado através do conhecido slogan "SPT/2000: saúde para todos até o ano 2000"; Na década de 1980-1990 as reformas neo-liberais ocorreram em conjuntura de ajustes macro-econômicos. Marcadas por uma ênfase na separação entre financiamento e prestação dos serviços, discurso de maior eficiência na gestão, estímulo a competição entre serviços públicos com a constituição de um "mercado interno" ou entre prestadores públicos e privados. São referências as mudanças implementadas no NHS da Inglaterra e um conjunto de medidas gerenciais introduzidas nos seguros privados americanos (managed care). A política de APS passa a ser divulgada por organismos internacionais (Banco Mundial) como uma estratégia seletiva orientada para grupos vulneráveis e excluídos do processo de produção; Final da década de 1990 - início século XXI: tem-se as reformas pró-coordenação.

A importância da integração entre os serviços substitui a ênfase na competição com retorno de um discurso sobre a organização de redes de cuidados coordenadas por uma atenção primária renovada. As transformações realizadas no NHS da Inglaterra com a chegada do partido trabalhista ao poder e documentos orientadores difundidos pela Organização Pan-americana de Saúde-OPAS são iniciativas que representam bem esse momento;

Período mais recente: A reforma americana conhecida como Obamacare institui o The Patient Protection and Affordable Care Act (PPACA - 2010) com implementação gradual da universalização da cobertura através de seguros privados. A crise européia impõe cortes orçamentários, reformas e novas pressões do mercado. O NHS volta a ser alvo de grandes transformações com redução das estruturas de gestão, estímulo a competição e prestação privada de serviços. O sistema nacional espanhol e português sofrem ajustes que podem ameaçar a legitimação social alcançada. É nesse contexto que surge a proposta de cobertura universal para países de média e baixa renda, divulgada no Relatório Mundial da Saúde em 2010(11).

\section{Avanço dos direitos e do acesso aos serviços na América Latina: a complexidade de superar a segmentação e a fragmentação dos serviços}

As reformas que ocorreram no continente latino-americano nas últimas décadas refletiram as conjunturas internacionais mencionadas, de tal modo que, é possível sugerir a hipótese de que o continente americano se constitui num grande laboratório de políticas de saúde e modelos assistenciais.

A ditadura de Pinochet introduziu seguradoras privadas (ISAPRES) com estímulo ao mercado, modelo adaptado e utilizado na Colômbia como forma de expandir a cobertura através de seguros subsidiados (Lei 100 de 1993), o que ficou conhecido como "pluralismo estruturado", com clara influência do momento neoliberal. No Brasil, a Constituição de 1988 reconheceu a saúde como um direito e a prestação universal de serviços integrais passou a ser realizada pelo Sistema Único de Saúde (SUS), um sistema público integrado sob financiamento fiscal. Tratase de uma reforma de caráter publicista cujo discurso correspondia ao momento "democrático-racionalizador" da conjuntura anterior, mas que terminou por ser implementada tardiamente numa conjuntura neoliberal e adversa.

Enfrentando mudanças de conjunturas e diversas transformações políticas internas, os três casos levaram a sistemas duais com uma complexa composição de serviços públicos e privados de difícil regulação e gestão que impõem desafios para superar a segmentação e a fragmentação que ainda predomina nesses sistemas.

No Brasil, houve uma expansão significativa da Estratégia da Saúde da Família (ESF) com medidas recentes na direção de melhoria do acesso e da qualidade. De um programa iniciado em 1994, essa política de atenção básica passa a ser considerada uma estratégia para reorientação do modelo assistencial e se constitui, por suas características e continuidade, em um exemplo importante de implementação de "antigas" e necessárias inovações na prestação de serviços.

As seguintes etapas podem ser identificadas ao longo de mais de duas décadas de sua existência: a primeira, de implantação, continuidade e superação de resistências diante da possibilidade de que pudesse representar uma APS seletiva; a segunda, de expansão e ajustes (incorporação de equipes de saúde bucal, criação de Núcleos de Apoio a Saúde da Família-NASFs, mudanças nos mecanismos de financiamento federal, estudos de avaliação) e, a terceira, que estava em andamento desde 2011 e que se caracterizava por atividades para melhoria do acesso e da qualidade (infra-estrutura, avaliação com pagamento por desempenho) ${ }^{(12)}$.

Neste contexto foi instituído o Programa Mais Médicos (PMM), através de Lei 12871 de 22 de outubro de 2013(13), com três componentes principais: provisão emergencial, ampliação da oferta de cursos, vagas e residências, novas diretrizes e parâmetros para a formação, incluindo a ênfase na atenção primária. 
Apesar de sua implantação recente, muitos trabalhos têm discutido esta iniciativa. Os resultados do provimento emergencial feito, principalmente, através de um acordo com o governo cubano, mostram melhoria do acesso e satisfação dos usuários. Houve diminuição de desigualdades por uma oferta de profissionais em municípios com populações vulneráveis e dificuldades em fixar médicos, além de melhores indicadores de produção e de práticas com maior responsabilização comunitária ${ }^{(14,15)}$. Mas, algumas preocupações têm sido debatidas e se referem à expansão do ensino médico em Escolas privadas, ausência de uma carreira para profissionais do SUS e o não enfrentamento de entraves estruturais do sistema de saúde como um todo (financiamento, novas formas de gestão, coordenação entre atenção básica e demais serviços) ${ }^{(15,16,17)}$. Em 2015, o número de especialistas em medicina de família e comunidade correspondia a $1,2 \%$ do total de especialistas brasileiros ${ }^{(18)}$ sendo a rotatividade de médicos e pessoal de enfermagem um problema antigo e considerado crítico $^{(19,20,21)}$.

Mudar as práticas é um processo longo havendo grande variação no grau de inovações que foram incorporadas aos processos de trabalho ${ }^{(22,23,24)}$. Um estudo sobre resultados do Programa de Melhoria do Acesso e Qualidade$P M A Q^{(25)}$ chama atenção para a expansão expressiva da ESF (em 2017, cobria em torno de $60 \%$ da população, com mais de 40.000 equipes implantadas) e, funcionando cada vez mais como entrada preferencial no sistema atendendo a uma demanda diversa. Existem dificuldades no acesso e na coordenação com a atenção especializada, o que coincide com observações feitas por outros autores ${ }^{(26,27)}$ que, também, discutem a necessidade de melhorar o cuidado clínico, diminuir o enfoque "programático" das práticas e aumentar o "prestígio" da atenção primária. Mas, tanto estes como outros estudos relevantes têm demonstrado resultados positivos da implantação dessa política com diminuição de desigualdades na utilização dos serviços ${ }^{(28)}$ na mortalidade em menores de cinco $\operatorname{anos}^{(29)}$ e nas internações por condições sensíveis a APS ${ }^{(30)}$.

O modelo de financiamento para a saúde no Brasil permanece contraditório com os princípios universalistas do Sistema Único de Saúde/SUS: em 2012 o gasto em saúde era elevado (9,3\% do PIB), porém, apenas 46,4\% dos fundos eram provenientes de fontes públicas ${ }^{(31,32)}$. Atualmente o sistema de saúde brasileiro é um sistema universal com uma cobertura duplicada e desigual: enquanto a população de renda mais baixa utiliza o SUS, usuários do segmento suplementar podem constitucionalmente recorrer aos dois, com direito a renúncia fiscal. A aprovação da Emenda Constitucional $n^{\circ} 95$ em dezembro de 2016 ${ }^{(33)}$ estabelecendo que despesas e investimentos públicos permaneçam limitados aos mesmos valores sendo apenas corrigidos pela inflação durante os próximos 20 anos, não apontam para mudanças positivas neste cenário.

No caso do Chile o processo de redemocratização permitiu reformas com a vigência de duas novas leis, a partir de 2005: a Ley de Autoridad Sanitaria e a Ley de Garantias Explícitas en Salud. Esta última merece destaque, pois representou uma inovação na tentativa de melhorar a macro-regulação de sistemas duais, sendo conhecida como Plan AUGE - Acceso Universal con Garantias Explícitas en Salud. O Plan AUGE define a cobertura obrigatória (garantias) dos principais problemas de saúde a serem atendidos tanto no sistema público como no privado. Foram selecionados problemas prioritários e estabelecidos parâmetros de acesso, oportunidade (tempo ou prazos para o atendimento), qualidade e proteção financeira (limites de co-pagamento ou gratuidade), que podem ser exigidos nos dois subsistemas. Atualmente, 80 patologias compõem a lista de garantias explícitas ${ }^{(34)}$.

Também ocorreram ações para ampliar o acesso, reorganizar e melhorar a qualidade dos serviços com aumento do aporte fiscal, diminuição de co-pagamentos, retirada de subsídios das ISAPRES, recuperação da rede pública, estímulo a APS e a organização de redes regionalizadas e integradas de serviços. Esses objetivos fazem parte de um extenso Plano de Metas divulgado pelo Ministério da Saúde para o período 2011-2020(35,36).

Um estudo ${ }^{(37)}$ comparou o sistema de saúde do Chile com o da Costa Rica, o qual sintetiza resultados de pesquisas sobre os efeitos do AUGE. Teria ocorrido um aumento na utilização de consultas gerando filas de espera e distribuição de "vouchers" para usuários do sistema público utilizarem prestadores privados; mas, também houve uma diminuição de internações por patologias incluídas na lista de garantias, o que sugere maior resolubilidade na atenção recebida. Há evidências de que problemas não incluídos no AUGE estão sendo menos atendidos havendo maiores tempo de espera para essas patologias. Permanecem desafios na diminuição de desigualdades: os mais ricos continuam a ter melhor acesso a especialistas, os pobres freqüentam mais a emergência, sem diferença significativa no uso de serviços de atenção primária.

Uma revisão ${ }^{(38)}$ destaca os esforços dessa medida para explicitar direitos e diminuir desigualdades na atenção, mas aponta fragilidades que se referem à metodologia de priorização dos problemas, divulgação junto aos mais vulneráveis, apoio dos profissionais, monitoramento e, limites dos recursos financeiros para garantir sua continuidade.

Em 2010, aproximadamente $74 \%$ da população chilena estava afiliada ao subsistema público, $17 \%$ a ISAPRES, $2 \%$ ao sistema de saúde das forças armadas e $7 \%$ não estava adscrita a nenhuma modalidade. A análise da 
composição do financiamento mostra que o aporte público oriundo de fontes fiscais com caráter redistributivo é ainda muito reduzido, sendo da ordem de 40\%. Em 2010 foram oferecidas 36 vagas para residência em medicina familiar com utilização de menos da metade, em 2011 foram 42 vagas com uma ocupação discretamente superior, o que pode ser considerado revelador dos resultados ainda discretos das políticas de estímulo a APS no país ${ }^{(39)}$.

As reflexões que emergem da análise do caso colombiano também não parecem demonstrar a superioridade de um modelo centrado em operadoras de seguros como estratégia para a universalização dos cuidados. No período de 1993 a 2003 a cobertura se expandiu de modo significativo alcançando $90 \%$ da população (38\% regime contributivo e $51 \%$ subsidiado). $O$ crescimento do plano subsidiado foi quase três vezes superior ao contributivo com uma diferença importante no rol de serviços prestados em cada um desses planos ${ }^{(40)}$.

Em 2010 uma grave crise institucional e financeira do Sistema General de Seguridad Social en Salud (SGSSS) levou a revisão da Ley 100 que o havia instituído com a votação de um novo marco legal em 2011 (Ley 1438). Um conjunto de problemas e diferentes interpretações foram referidos para explicar essa situação: fenômeno crescente de judicialização (tutelas), consumo desregulado de medicamentos, ausência de prioridades sociais, insuficiência de recursos públicos, problemas econômicos estruturais com maior incremento do regime subsidiado frente ao contributivo, questões administrativas de diversas ordens (falta de clareza e atualização nas listas de garantias, por exemplo), corrupção com perda de legitimidade das Empresas Promotoras e Salud (operadoras dos seguros) e baixa efetividade do sistema ${ }^{(40,41)}$.

Essa conjuntura foi marcada por um intenso debate entre autoridades governamentais, políticos, entidades profissionais e sociedade civil acerca dos resultados, impasses e possibilidades de aperfeiçoamento do sistema colombiano. A nova lei estabelece o acesso universal como um direito da cidadania e a atenção primária como estratégia para organizar a prestação dos serviços. Para alcançar esse propósito o novo marco legal prevê a unificação dos planos de benefícios com uma atualização a cada dois anos dos procedimentos cobertos. Em 2012, a arquitetura do sistema continuava composta pelos regimes contributivo, subsidiado, especial (militares, magistério, petroleiros, entre outros) e, por não assegurados cujo atendimento é previsto em serviços disponíveis das Secretarias de Saúde dos entes territoriais (Departamentos e municípios) ${ }^{(40,41)}$.

O processo de equidade do Plano Obrigatório de Saúde já havia iniciado em 2009, primeiramente com a população abaixo de 18 anos, em seguida num projeto piloto (Barranquilla e Cartagena) e, por último com a inclusão do grupo etário acima de 60 anos ( $17 \%$ da população). A previsão governamental é de que esse processo se estenda de modo progressivo aos grupos populacionais de 18 a 35 anos e de 35 a 60 anos $^{(42)}$.

Os resultados obtidos pelo modelo colombiano permanecem controversos. Para alguns, expandiu o acesso, reduziu desigualdades e melhorou indicadores de saúde, além de ter catalisado a discussão sobre direitos e garantias em saúde ${ }^{(8,4)}$. Em 1993, o gasto direto das famílias representava $49 \%$ do gasto total em saúde, em 2010 passou para $7 \%$. Nos segmentos mais pobres da população, a não atenção por um problema de saúde caiu de $33,2 \%$ para 1,3\% (1993- 2010), tendo dobrado a cobertura de atenção pré-natal (1990-2010) ${ }^{(40)}$.

Para outros, o modelo mantém desigualdades, além de representar uma abertura para o mercado privado com precarização do trabalho e uma fragmentação difícil de regular ${ }^{(41,43)}$. A análise de dados da Encuesta Nacional de Calidad de Vida nos anos 2003 e 2008 mostram diferenças importantes entre os dois tipos de regimes com a existência de altas coberturas para cuidados curativos, ocorrendo o contrário com os serviços preventivos ${ }^{(42)}$. Autores $^{(44-46)}$ consideram que a menção à APS e a organização de redes integradas na nova Lei se constituem em apropriações retóricas de objetivos defendidos para sistemas universais, uma vez que não foram introduzidas mudanças estruturais que permitam viabilizá-las. Mesmo que a atenção primária seja considerada como uma estratégia para orientar a organização e a prestação de serviços, existem dúvidas quanto ao real alcance dessa intenção normativa. Os objetivos do marco legal estariam direcionados para uma maior regulação das operadoras e sustentabilidade financeira do sistema sem uma mudança de suas características de modo a favorecer a efetiva implementação desta "antiga inovação"(44).

\section{As inovações nos sistemas nacionais de saúde orientados para atenção primária em Espanha e Portugal}

Espanha e Portugal implementaram sistemas nacionais de saúde inspirados no modelo britânico mas, diferentemente deste, a porta de entrada é constituída por centros de saúde com profissionais que fazem parte do sistema público. Surgiram nos contextos de democratização que marcaram as décadas de 1970 e 1980 nesses países. O sistema é descentralizado na Espanha para as Comunidades autônomas - CCAA e, em Portugal para regiões. Em ambos houve um desenvolvimento muito importante da atenção primária à saúde. 
$\mathrm{Na}$ Espanha, os recursos fiscais para financiamento do Sistema Nacional de Salud são transferidos pelo governo central para as Comunidades Autônomas que se responsabilizam pela organização, gestão e prestação dos serviços. A propriedade e oferta dos serviços nos sistemas regionais é predominantemente pública, sendo a gestão realizada por uma Consejeria de Salud. Esses sistemas possuem entidade jurídica própria através de diversas fórmulas: organismos administrativos autônomos similares a administração pública (quase todas as CCAA), entes públicos de direito privado (P. Basco, Madrid), empresa pública (Instituto Catalan de Salud - ICS de Catalunha).

Na maior parte dos sistemas regionais, a prestação de serviços se divide em atenção primária (porta de entrada) e cuidados especializados sendo organizada por Áreas de Saúde (100.000-200.000 hab.), com um Hospital Geral para emergência, internações e referência especializada. Há estruturas de gerências em cada Área com um grau variável de integração entre os dois níveis de cuidados e as ações de saúde pública. As Áreas dividem-se em Zonas Básicas e a população adscrita varia de 5.000 a 25.000 habitantes.

Até 2012 o acesso, com portabilidade nacional, era garantido a todos os espanhóis ou residentes legais bastando inscrever-se na lista de um médico de APS de um Centro de Saúde (medico de cabecera), cujo nome passava a constar no cartão do usuário. Recentemente, o contexto de recessão econômica determinou medidas de ajustes com mudanças legais que alteraram os princípios constitutivos do SNS (Decreto Real Lei 16/2012)(47). A saúde deixou de ser um direito de cidadania voltando a estar vinculado à condição de beneficiário da Seguridade Social, o que pode vir a ameaçar a universalização e a solidariedade intergeracional.

Foi o Decreto de Estruturas Básicas de Saúde ${ }^{(48)}$ promulgado antes mesmo da Ley General de Sanidad - LGS que iniciou a reforma de atenção primária mediante a criação de Centros de Saúde, fortemente influenciada por experiências internacionais que haviam ocorrido na década anterior, conforme já assinalamos. Um acontecimento importante desse momento foi a criação, pelo Ministério da Saúde, da especialidade de Medicina de Família e Comunidade $(M F y C$ ) dentro do sistema centralizado de formação de especialistas conhecido como MIR (médicos internos y residentes).

A formação incremental desses profissionais ( 500 vagas por ano) foi uma das principais inovações realizadas no processo de construção do SNS espanhol sendo imprescindível destacá-la. Este movimento favoreceu mudanças nos serviços, ao qual se somaram os profissionais de saúde pública. Uma parte desse grupo, ao ingressar em cargos de direção do novo governo democrático foi responsável pela reforma da Atenção Primária.

O Ministério mantém a responsabilidade na formação e especialização dos profissionais através do sistema nacional de residências (MIR), com definição e publicação do número de vagas, acreditação dos programas de ensino o que é feito anualmente em conjunto com as Sociedades Científicas. Porém, mesmo na Espanha cujo modelo assistencial está organizado a partir da APS, há um importante déficit de médicos de família e comunidade, o que mostra a complexidade da questão e a necessidade de enfrentá-la através de diversas medidas (melhores incentivos, maior autonomia, aumento do prestígio da especialidade). Tomando-se como referência o atual padrão de preenchimento de vagas, seria necessário de 8 a 9 cohortes de residentes para suprir esse déficit, enquanto que a maior parte das especialidades cobriria seu déficit com um só concurso (anestesia, cirurgia vascular, cardiologia, cirurgia geral, traumatologia, dermatologia, neurologia, ginecologia e obstetrícia, radiologia e reumatologia) ${ }^{(49)}$.

Em 2011, apesar de problemas relacionados ao crescimento de filas de espera para internações e exames especializados, o desempenho do SNS era avaliado de modo favorável por profissionais, gestores e população( ${ }^{(50)}$. Em 2009, 21\% dos espanhóis consideravam que o sistema funcionava bastante bem e $48 \%$ que funcionava bem, mas necessitava de mudanças, conforme dados do Barômetro Sanitário (inquérito de opinião realizado anualmente pelo Ministério da Saúde). Para a maioria o sistema público tem melhor qualidade e, no caso de uma doença grave $58 \%$ dos entrevistados elegeriam esses serviços. Os cuidados de atenção primária eram os que melhor pontuavam em termos de satisfação.

Para a Federación de las Asociaciones para la Defensa de la Sanidad Publica as medidas restritivas que vem sendo tomadas como resposta a crise econômica (cortes nos gastos públicos, redução de pessoal, fechamento de leitos, aumento de co-pagamentos e do gasto direto das famílias) comprometem a qualidade e equidade do SNS e dos indicadores de saúde, influenciando de modo negativo estas avaliações. De 2009 a 2015, houve um incremento de $47 \%$ nas listas de espera cirúrgicas. Estes fatos ameaçam a legitimidade do sistema público favorecendo um crescente processo de privatização que pressionaria o modelo assistencial para um maior consumo de medicamentos e incorporação tecnológica(51).

Um documento do Ministerio de Sanidad, Servicios Sociales e lgualda ${ }^{(52)}$ sintetiza séries históricas do Barômetro Sanitário. Entre 2002-2016 houve um aumento importante no porcentual de entrevistados que consideram ter ocorrido piora nos serviços de APS, atenção especializada, hospitais e listas de espera. Entretanto, é interessante assinalar 
que a aprovação do sistema nacional ainda não se modificou de modo negativo: a "nota" atribuída pelos usuários passou 5,94 para 6,55 neste período. Em 2016, em torno de 20\% consideravam que o SNS funcionava bem, $46 \%$ que necessitava algumas mudanças e, menos de $5 \%$ que "estava tão mal que seria necessário refazê-lo".

O SNS português foi instituído em 1979 e, em 1982 foi criada a carreira de clinica geral. Este é um dado interessante que demonstra o compromisso com esse modelo de atenção nos primórdios de sua construção. Mas, no caso português a inovação que consideramos mais interessante destacar é a reforma dos cuidados primários implementada a partir de $2005^{(53-55)}$.

Essa reforma é fruto de uma trajetória de expansão e adequação dos cuidados primários de saúde/CSP que inicia com Centros de Saúde denominados de $1^{\text {a }}$ e $2^{\text {a }}$ Geração, criados no início da década de 1970 e 1980 . É nesse momento que se consolida a ampliação do sistema de saúde português com uma direção para a atenção primária ${ }^{(56)}$. Gradativamente as prioridades tornam-se outras e o CSP perdem protagonismo, apoio político e financeiro.

Em 1995, uma nova reforma propõe mudanças encontrando forte oposição, mas propostas teóricas e experiências inovadoras subsistem e irão se constituir em referências importantes. Em 1991, a Associação Portuguesa de Médicos de Clínica Geral havia publicado um documento sobre o futuro da medicina de família no país e, em 1998 - Ministério da Saúde implantou um regime remuneratório experimental em vinte unidades de saúde semelhante ao modelo proposto por essa Associação. A avaliação positiva dessa experiência levou a recomendação de que fosse expandida a todos os Centros.

Em 2005 foi constituída a Missão para os Cuidados em Saúde Primários/ MCSP que deveria conduzir um projeto global de reconfiguração dos Centros de Saúde com implementação das Unidades de Saúde Familiar/USF. Uma das principais características desse processo foi o de impulsionar um movimento "de baixo para cima" promovendo a auto-organização dos profissionais de saúde através de um processo de candidaturas voluntárias para a constituição das equipes (médico de família, enfermeiros e secretários clínicos). Essas candidaturas eram objetos de avaliação técnica devendo cumprir com uma série de requisitos. A cobertura populacional prevista situou-se entre 4.000 a 16.000 pessoas, correspondendo a uma lista de usuários de aproximadamente três a dez médicos de família, com alguma flexibilidade tanto no numero de enfermeiros como de administrativos.

Essa nova forma de prestar serviços começou a se constituir a partir de uma rede formada por pequenas equipes multiprofissionais, descentralizadas, voluntárias e dotadas de considerável grau de autonomia contratualizada ${ }^{(55)}$. O autor destaca aspectos que considera inovadores e centrais na reforma portuguesa: candidatura voluntária ("equipes que se escolhem"), autonomia organizativa, funcional e técnica, inter-substituição, contratualização de uma carteira básica de serviços, plano de ação com metas anuais, critérios explícitos de avaliação e prestação de contas e, um sistema de remuneração sensível à carga de trabalho e ao desempenho (acessibilidade, qualidade, eficiência, satisfação e resultados), adequado a cada categoria profissional. Uma parte dos incentivos recebidos pode ser revertida para melhorias na própria Unidade.

Foram previstos dois estágios evolutivos na constituição das USFs que se diferenciam em função do grau de autonomia, modalidades de remuneração, financiamento e estatuto jurídico. O modelo " $A$ " é uma fase de aprendizagem e aperfeiçoamento e o " $\mathrm{B}$ " é previsto para as equipes com maior amadurecimento organizacional e um processo de contratualização de desempenho mais exigente. Em 2016, estavam em atividade um total de 459 USFs (227 correspondem ao modelo B e 232 ao A) com uma cobertura que atinge a aproximadamente $50 \%$ dos habitantes do país ${ }^{(56)}$.

Nesse processo de reforma foram também constituídas novas unidades de gestão que são os Agrupamentos dos Centros de Saúde (ACeS). Além das USFs, fazem parte do âmbito de atividade dessas estruturas as Unidades de Cuidados de Saúde Personalizados- UCSP (os centros de saúde tradicionais), de Cuidados na Comunidade, de Saúde Pública e de Recursos Assistenciais Partilhados ${ }^{(56)}$.

Um estudo de avaliação da implantação da reforma da saúde familiar em Portugal identificando como principais avanços a acessibilidade, o trabalho em equipe, a qualidade dos cuidados, inovações na gestão, nas condições de trabalho e a infraestrutura(53). Mostram que os principais desafios são a integração com especialidades, indefinições político institucionais, informatização, integração nos centros de saúde e organização dos processos de trabalho. Esses achados coincidem com resultados de outros estudos sobre o tema ${ }^{(54,55)}$ e com dados publicados nos Relatórios da Primavera do Observatório Português de Sistemas de Saúde ${ }^{(57)}$.

Em 2016, uma pesquisa realizada por uma instituição pública portuguesa encarregada de monitorar os serviços (Entidade Reguladora da Saúde) demonstrou um melhor desempenho das USFs na maioria dos indicadores considerados (utilização, tempos máximos de respostas garantidos, vínculo, promoção, prevenção, internações por condições sensíveis, eficiência). Este trabalho mostra de modo contundente o que o autor denomina de "valor 
da mudança”(58). Apesar dessas evidências, a reforma foi interrompida durante a conjuntura recessiva enfrentada pelo país, voltando a ser prioritária a partir de 2016 com a divulgação de um Plano Estratégico e Operacional pelo Ministério da Saúde (Coordenação para os Cuidados Primários) ${ }^{(59)}$.

\section{Desafios para os sistemas contemporâneos e a importância de "antigas e jovens inovações" para a garantia de um acesso oportuno aos serviços de saúde}

Conforme já apontamos em trabalho anterior ${ }^{(60)}$, a análise comparada das reformas na saúde revela um movimento quase pendular em que muitos discursos se repetem. Em momento de crises e ajustes macroeconômicos, o pêndulo oscila para a contenção de gastos, supressão de direitos e abertura ao mercado sob argumento de que a competição garante melhor qualidade. Em conjunturas econômicas favoráveis ou aberturas políticas, a saúde é vista como condição para o desenvolvimento e coesão social, com ênfase na construção de redes coordenadas pela atenção primária para garantir a racionalidade necessária à qualidade e sustentabilidade dos sistemas.

A perspectiva comparada tem permitido identificar alguns desafios comuns aos sistemas contemporâneos ${ }^{(61)}$. Primeiramente, existe um grupo de questões macroestruturais, algumas das quais extrapolam o setor saúde mas tem uma influência direta sobre ele: o modelo de desenvolvimento econômico, as opções políticas e financeiras frente a proteção social, o complexo produtivo da saúde, a articulação público-privado no financiamento, na prestação e na utilização dos serviços e, a transição demográfica e epidemiológica das populações.

Além disso, qualquer análise dos limites e das possibilidades na introdução de "antigas ou jovens inovações" impõe uma análise da dinâmica de acumulação setorial. Ao complexo médico-industrial (tecnologia e indústria farmacêutica) soma-se hoje um complexo médico-financeiro (seguros privados), com hipertrofia do setor de serviços (assessorias, informação, exportação de hospitais, turismo médico ${ }^{(62)}$. Existiriam ganhos complementares entre esses segmentos levando a uma inflação crônica com crescimento dos gastos setoriais. A capacidade de regulação pública torna-se reduzida diante de uma complexa pluralidade de agentes relacionando-se com a política industrial, financeira e de serviços pessoais, todas permeadas por valores e normas éticas referentes à intervenção sobre a vida humana ${ }^{(63)}$.

As estimativas de crescimento do mercado de serviços de saúde para 2014 apontam "oportunidades" na Ásia, nas economias em transição, África e, na América Latina ${ }^{(64)}$. Isto significa que a disputa de interesses pode se tornar ainda mais acirrada e, sem mudanças no modelo assistencial, a universalização dos cuidados poderá vir a representar um aumento desregulado de consumo sem correspondência com as necessidades de saúde da população.

A descrição do caso espanhol e português mostra que sistemas universais sob controle público são viáveis e sustentáveis do ponto de vista macroeconômico. A prestação integrada de serviços de base territorial orientada pelos princípios da atenção primária favorece o bom desempenho desses sistemas. Uma das maiores contribuições dessa política de expansão de garantias e direitos na saúde é ter inovado no plano das representações sociais ao obterem avaliações positivas que legitimam esse modelo de atenção. Este fato se tornou evidente tendo em vista o importante papel desempenhado pelos movimentos sociais que, em aliança com outras forças sociais, impuseram limites ou mesmo reverteram medidas adversas ao SNS implementadas durante a crise européia ${ }^{(" 51)}$.

\section{CONCLUSÃO}

É importante ter clareza de que há um confronto entre concepções acerca do modo de organizar e prestar serviços. De um lado um modelo que visa garantir acesso oportuno e, de outro, uma tendência que tende a pressionar na direção de oportunidades de negócios. A construção de sistemas universais na América Latina não deve significar apenas expansão da cobertura, mas um esforço na direção da primeira possibilidade. Antigas e jovens inovações serão, portanto, necessárias e bem-vindas.

Por essa razão, "antigas e jovens inovações" terão um papel importante para fazer frente a esse desafio ao promoverem um acesso oportuno aos serviços. As seguintes questões do âmbito da meso e da microgestão tornamse centrais: a constituição de redes integradas, o uso racional de tecnologias e medicamentos, novas formas de gestão com maior autonomia, co-responsabilização e modalidades de pagamento por desempenho, o processo de trabalho em APS (equipe multidisciplinar, interação entre generalistas e especialistas, o cuidado de doenças crônicas), mas principalmente a valorização e a formação desses profissionais. Um dos grandes desafios da "antiga inovação" representada pela atenção primária é de que essas práticas necessitam responder de modo adequado as situações de doença e, ao mesmo tempo, desenvolver ações para evitá-las e promover a saúde.

No entanto, o caráter público que favorece virtudes encerra também fragilidades havendo problemas de eficiência microeconômica. A existência de listas de espera aparece como um dos problemas mais importantes dos sistemas 
nacionais. E, sabe-se que a forma como a qualidade é percebida pelos usuários influencia no crescimento de seguros privados podendo ameaçar sua sustentação política e social, ao longo do tempo. Qualidade e equidade são atributos que estão juntos e se retroalimentam, a evasão das classes médias é prejudicial ao afastar do sistema segmentos com maior poder de pressão e influência.

Os modelos que apostaram na prestação privada dos serviços e numa dinâmica de mercado, como foi o caso do Chile e da Colômbia, não demonstraram ser uma alternativa superior ao modelo de sistemas nacionais. A experiência brasileira de organização do SUS com sua orientação para a APS mostra ser necessário coerência entre diretrizes constitucionais, financiamento, modelo de gestão, formação de profissionais, bem como, sua adequada inserção num sistema público valorizado. Reverter representações sociais que se tornaram adversas à noção de serviços públicos e obter legitimação social para este tipo de política de saúde exige medidas efetivas desta ordem.

Fonte financiadora: Ministério da Saúde, Secretaria de Gestão Estratégica e Participativa e Conselho Nacional de Secretarias Municipais de Saúde.

\section{REFERÊNCIAS}

1. Observatório Português de Sistemas de Saúde. Relatório de Primavera 2008 - Sistema de Saúde Português: riscos e incertezas [Internet]. Respostas inovadoras em saúde 2008, 71-90. [acesso em 2017 Mai 10]. Disponível em: http://www.opss.pt/sites/opss.pt/relatorios/relatorio-primavera-2008/

2. Macinko J, Starfield B, Shi L. The contribution of primary care systems to health outcomes within Organization for Economic Cooperation and Development (OECD) countries, 1970-1998. Health Serv Res. 2003;38(3):83165.

3. Normam $A H$, Tesser $C D$. Prevenção quaternária na atenção primária à saúde: uma necessidade do Sistema Único de Saúde. Cad Saúde Pública. 2009;25(9):2012-20.

4. Terris M. Tendencias y perspectivas de los tres sistemas mundiales de atención médica. Foro Mund Salud. 1980;93-103.

5. Fleury S, Ouverney AM. Política de saúde: uma política social. In: Giovanella L, Escorel S, Lobato LVC, Noronha JC, Carvalho Al, organizadores. Políticas e sistema de saúde no Brasil. Rio de Janeiro: Editora Fiocruz; 2012. p. 25-57.

6. Conill EM. Sistemas comparados de saúde. In: Campos GVS, Bonfim JR, Minayo MCS, Akerman M, Drumond M Jr, Carvalho YM, organizadores. Tratado de Saúde Coletiva. São Paulo: Fiocruz; 2012. p. 591-659.

7. Freire Campo JM. Los sistemas de aseguramiento sanitario de riesgos de enfermedad en España. Derecho y Salud. 2007;15:41-60.

8. Giovanella L, Feo O, Faria M, Tobar S. Sistemas de salud en Suramerica: desafíos para a la universidad la integralidad y la equidad. Rio de Janeiro: Instituto Suramericano de Gobierno en Salud; 2012.

9. Sojo A. Condiciones para el acceso universal a la salud en América Latina: derechos sociales, protección social y restricciones financieras y políticas. Ciênc Saúde Colet. 2011;16(6):2673-85.

10. Piola S. Relatório técnico de análise da matriz de indicadores do Observatório Iberoamericano de Políticas e Sistemas de Saúde. Brasília: OIAPS; 2015.

11. Organización Mundial de la Salud. La financiación de los sistemas de salud. El camino hacia la cobertura universal [Internet]. Ginebra: OMS; 2010. [acesso em 2017 Mai 15]. Disponível em: http://apps.who.int/iris/bits tream/10665/44373/1/9789243564029_spa.pdf.

12. Brasil. Portaria n. 1.654, de 19 de julho de 2011. Institui, no âmbito do Sistema Único de Saúde, o Programa Nacional de Melhoria do Acesso e da Qualidade da Atenção Básica (PMAQ-AB) e o Incentivo Financeiro do PMAQ - AB, denominado Componente de Qualidade do Piso de Atenção Básica Variável - PAB Variável. Diário Oficial da República Federativa do Brasil, Brasília. 2011.

13. Brasil. Lei 12.871, de 22 de outubro de 2013. Institui o Programa Mais Médicos, altera as Leis n.8.745, de 9 de dezembro de 1993, e n.6.932, de 7 de julho de 1981, e dá outras providências. Diário Oficial da República Federativa do Brasil, Brasília. 2013. 
14. Fachini LA, Sandro RB, Silva AG Jr, Giovanella L. O Programa mais médicos: análises e perspectivas. Ciênc Saúde Colet. 2016;21(9):2652.

15. Campos GWS, Pereira N Jr. Atenção primária e o Programa Mais Médicos do Sistema Único de Saúde: conquistas e limites. Ciênc Saúde Colet. 2016;21(9):2655-63.

16. Scheffer M. Para muito além do Programa Mais Médicos. Ciênc Saúde Colet. 2016;21(9):2664-66.

17. Trindade TG, Batista SR. Medicina de Família e Comunidade: agora mais do que nunca!. Ciênc Saúde Colet. 2016;21(9):2667-69.

18. Scheffer M, coordenador. Demografia Médica no Brasil 2015. São Paulo: Conselho Federal de Medicina; 2015.

19. Ney MS, Rodrigues PHA. Fatores críticos para a fixação do médico na Estratégia Saúde da Família. Physis. 2012;22(4):1293-1311.

20. Benning EL, Da Guarda FRB. Análise da estratégia de saúde da família em Pernambuco com base no tempo médio de permanência dos profissionais médicos e de enfermagem, no período de 2001 a 2006 [trabalho de conclusão de curso]. Pernambuco: Fundação Oswaldo Cruz; 2006.

21. Perpétuo IHO, Oliveira AC, Ribeiro MM, Rodrigues RB. A categoria profissional dos médicos: fatores condicionantes de sua atração e fixação na Atenção Primária a Saúde em Minas Gerais. Belo Horizonte: Observatório de Recursos Humanos em Saúde; 2009.

22. Almeida PF, Giovanella L, Nunan BA. Coordenação dos cuidados em saúde pela atenção primária à saúde e suas implicações para a satisfação dos usuários. Saúde Debate. 2012;36(94):375-91.

23. Cunha EM, Giovanella L. Longitudinalidade/continuidade do cuidado: identificando dimensões e variáveis para a avaliação da Atenção Primária no contexto do sistema público de saúde brasileiro. Ciênc Saúde Colet. 2011;16 (Suppl 1):1029-42.

24. Mendonça MHM, Martins MIC, Giovanella L, Escorel L. Desafios para gestão do trabalho a partir de experiências exitosas de expansão da Estratégia de Saúde da Família. Ciênc Saúde Colet. 2010;15(5):2355-65.

25. Fausto MCR, Giovanella L, Mendonça MHM, Seidl H, Gagno J. A posição da Estratégia Saúde da Família na rede de atenção à saúde na perspectiva das equipes e usuários participantes do PMAQ-AB. Saúde Debate. 2014;38(spe):13-33.

26. Gérvas J, Fernández MP. É possível transformar o círculo vicioso de má qualidade em um círculo virtuoso de boa qualidade no trabalho clínico e comunitário na atenção primária no Brasil [Internet]. Rio de Janeiro: SBMFC. 2015; [acesso 2017 Mai 15]. Disponível em: http://www.sbmfc.org.br/default.asp?site_Acao=MostraPagina\&P aginald $=524$.

27. Conill EM. Debatedores. Sobre a necessidade de legitimar as práticas de atenção primária. Saúde Debate. 2012;36(94):355-7.

28. Macinko J, Lima-Costa MF. Horizontal equity in health care utilization in Brazil, 1998-2008. Int J Equity Health. 2012;11:33.

29. Rasella D, Aquino R, Barreto ML. Reducing childhood mortality from diarrhea and lower respiratory tract infections in Brazil. Pediatrics. 2010;126(3):e534-40.

30. Mendonça CS, Harzheim E, Duncan BB, Nunes LN, Leyh W. Trends in hospitalizations for primary care sensitive conditions following the implementation of Family Health Teams in Belo Horizonte, Brazil. Health Policy Plan. 2012;27(4):348-55.

31. Observatório Iberoamericano de Políticas e Sistemas de Saúde. Matriz de indicadores [Internet]. 2017; [acesso 2017 Mai 17]. Disponível em: http://oiapss.icict.fiocruz.br/matriz.php?ling=2.

32. Machado FG. Os Gastos públicos e privados com saúde de 2000 a 2015 [monografia] [Internet]. Florianópolis: Universidade Federal de Santa Catarina, 2015; [acesso 2017 Mai 16]. Disponível em: https://repositorio.ufsc. br/bitstream/handle/123456789/158347/Monografia\%20do\%20Felipe\%20Galv\%C3\%A3o.pdf?sequence=1

33. Brasil. Emenda Constitucional n.95, de 15 de dezembro de 2016. Altera o Ato das Disposições Constitucionais Transitórias, para instituir o Novo Regime Fiscal, e dá outras providências. Diário Oficial da República Federativa do Brasil, Brasília. 2016. 
34. Chile. Superintendencia de Salud. Garantías Explicitas en Salud (AUGE o GES) [Internet]. 2017; [acesso 2017 Mai 17]. Disponível em: http://www.supersalud.gob.cl/difusion/665/w3-propertyvalue-1962.html\#acordeonAuge

35. IRD EUROsocial Salud. Dossiers nacionales de atención primaria en salud y integración con otros niveles de atención. Intercambio III. Rio de Janeiro: Fiocruz; 2007.

36. Gobierno de Chile. Metas 2011-2020: Elige Viver Sano. [Internet]. 2010; [acesso 2017 Mai 02]. Disponível em: http://www.minsal.cl/portal/url/item/c4034eddbc96ca6de0400101640159b8.pdf

37. Hernández LO, Salgado DP. Chile and Costa Rica: different roads to universal health in Latin America [Internet]. Occasional Paper. 2014;23. [acesso em 2017 Mai 18]. Disponível em: http://www.municipalservicesproject.org/ publications/chile-and-costa-rica-different-roads-universal-health-latin-america.

38. Missoni E, Solimano G. Towards Universal Health Coverage: the Chilean experience [Internet]. Genebra: WHO; 2010. [acesso em 2017 mai 18]. Disponível em: http://www.who.int/healthsystems/topics/financing/ healthreport/4Chile.pdf

39. Giovanella L, Feo O, Faria M, Tobar S. Sistemas de salud en Suramerica: desafíos para a la universidad la integralidad y la equidad. Rio de Janeiro: Instituto Suramericano de Gobierno en Salud; 2012. Sistema de Salud en Chile; p. 298-347.

40. Calderón CAA, Botero JC, Bolaños JO, Martínez RR. Sistema de salud en Colombia: 20 años de logros y problemas. Ciênc Saúde Colet. 2011;16(6):2817-28.

41. Romero RV. Reducción de los derechos sociales. Ciênc Saúde Colet. 2011;16(6): 2686-96.

42. Giovanella L, Feo O, Faria M, Tobar S. Sistemas de salud en Suramerica: desafíos para a la universidad la integralidad y la equidad. Rio de Janeiro: Instituto Suramericano de Gobierno en Salud; 2012. Sistemas de salud en Colombia; p. 348-439.

43. Homedes N, Ugalde A. Reformas de salud y equidad en América Latina: Health reform and equity in Latin America. Ciênc Saúde Colet. 2011; 16(6):2686-96.

44. Franco-Giraldo A. Referentes teóricos para el análisis de la reforma del sistema de salud colombiano. Rev Gerenc Polit Salud [Internet]. 2012 [acesso 2017 Mai 10];11(22):28-42. Disponível em: http://www.scielo.org. $\mathrm{co/pdf/rgps/v11n22/v11n22a03.pdf.}$

45. Franco-Giraldo A. La última reforma del sistema general de seguridad social en salud Colombiano. Rev Salud Pública [Internet]. 2012 [acesso 2017 Mai 19];14(5):865-77. 2012. Disponível em: http://www.scielosp.org/pdf/ rsap/v14n5/v14n5a13.pdf.

46. Casallas AL. Seminario El impacto de la crisis en los sistemas sanitarios de Iberoamérica [Internet]. 2016; [acesso 2017 Mai 10]. Disponível em: http://www.oiapss.org/wp-content/uploads/2017/01/El-impacto-de-la-crisis-en-lossistemas-sanitarios-de-lberoam\%C3\%A9rica_ANA-LUCIA-CASSALAS.pdf.

47. Gobierno de España. Real Decreto-ley 16/2012, de 20 de abril, de medidas urgentes para garantizar la sostenibilidad del Sistema Nacional de Salud y mejorar la calidad y seguridad de sus prestaciones. Boletín Oficial Del Estado n. 98, de 24 de abril de 2012.

48. Gobierno de España. Real Decreto-ley 137/1984, de 11 de enero. Dispõe sobre Estructuras Básicas de Salud. Boletín Oficial Del Estado n. 27, de 21 de fevereiro de 1984.

49. Pérez PB, López-Valcárcel BG. Oferta y necesidad de especialistas médicos en España (2008-2025). Madrid (ES): Universidad de Las Palmas de Gran Canaria; 2009.

50. Conill EM, Giovanella L, Almeida PF. Listas de espera em sistemas públicos: da expansão da oferta para um acesso oportuno? Considerações a partir do Sistema Nacional de Saúde espanhol. Ciênc Saúde Colet. 2011;16(6):2783-94.

51. Garcia MM. Privatización de la Sanidad Pública y sus consecuencias [Internet]. 2016; [acesso 2017 Mai 22]. Disponível em: http://www.oiapss.org/wp-content/uploads/2017/01/Privatizaci\%C3\%B3n-de-la-SanidadP\%C3\%BAblica-y-sus-consecuencias_MANUEL-MARTIN-GARCIA.pdf 
52. Gobierno de España. Ministerio de Sanidad, Servicios Sociales e Igualdad. Barometro Sanitario 1995-2016 [Internet].1994; [acesso 2017 Mai 22]. Disponível em: https://www.msssi.gob.es/estadEstudios/estadisticas/ BarometroSanitario/Barom_Sanit_2016/BS_1995_2016_Princ_var_CCAA.pdf.

53. Rocha PM, Sá AB. Reforma da Saúde Familiar em Portugal: avaliação da implantação. Ciênc Saúde Colet. 2011;16(6):2853-63.

54. Biscaia A. Futurar em positivo: satisfação no trabalho. Portugal: Grafisol; 2013.

55. Botelho H. Formas e estratégias de remuneração dos profissionais da APS em Portugal. In: Observatório Iberoamericano de Políticas e Sistemas de Saúde. Seminário Internacional: a formação e a remuneração dos profissionais responsáveis pela atenção primária em saúde. Brasília: Conselho Nacional de Secretários de Saúde - CONASS; 2014.

56. Biscaia AR, Heleno LCV. A Reforma dos cuidados de saúde primários em Portugal: portuguesa, moderna e inovadora. Ciênc. Saúde Colet. [Internet]. 2017 [acesso 2017 Mai 22];22(3):701-12. Disponível em: http://www. scielo.br/scielo.php?script=sci_arttext\&pid=S1413-81232017002300701\&lng=pt\&nrm=iso\&tlng=pt

57. Observatório Português de Sistemas de Saúde. Relatório Primavera 2011: da depressão da crise para a governação prospectiva da saúde [Internet]. Coimbra (PT): Observatório Português de Sistemas de Saúde; 2011. [acesso 2017 Mai 23]. Disponível em http://www.observaport.org/sites/observaport.org/files/RelatorioPrimavera2011_OPSS1. pdf

58. Entidade Reguladora da saúde. Estudo sobre as Unidades de Saúde Familiar e as Unidades de Cuidados de Saúde Personalizados [Internet]. Porto: ERS; 2016. [acesso 2017 Mai 23]. Disponível em: https://www.ers.pt/ uploads/writer_file/document/1793/ERS_-_Estudo_USF_e_UCSP_-_final_v.2_.pdf.

59. República Portuguesa. Serviço Nacional da Saúde. Plano Estratégico e Operacional [Internet]. 2016 [acesso 2017 Mai 23]. Disponível em: https://www.sns.gov.pt/wp-content/uploads/2016/02/Plano_Estrategico_e_Operacional. pdf

60. Conill, EM. A importância da continuidade dos sistemas nacionais europeus para as políticas de saúde na América Latina. Cad Saúde Pública. 2014;30(11):2253-55.

61. Conill EM, Freire JM, Giovanella L. Desafios para a consolidação dos sistemas de saúde ibero-americanos: temáticas que a análise comparada permite circunscrever. Ciênc Saúde Colet. 2011;16(6):2670.

62. Andreazzi MFS. Kornis GEM. Padrões de acumulação setorial: finanças e serviços nas transformações contemporâneas da saúde. Ciênc Saúde Colet. 2008;13(5):1409-20.

63. Deloitte. Global health care outlook Shared challenges, shared opportunities [Internet]. 2014 [acesso 2017 Mai 23]. Disponível em: file:///C:/Users/dell/Downloads/dttl-Ishc-2014-global-health-care-sector-report.pdf

64. Giovanella L, Stegmuller K. The financial crisis and health care systems in Europe: universal care under threat? Trends in health sector reforms in Germany, the United Kingdom, and Spain. Cad Saúde Pública. 2014;30 (11):2263-81.

\section{Endereço para correspondência:}

Eleonor Minho Conill

Rua Vento Sul, 306

CEP: 88063-070 - Florianópolis - SC - Brasil

E-mail: eleonorconill@gmail.com 